La

Révolution

française

\section{La Révolution française}

Cahiers de l'Institut d'histoire de la Révolution française

\section{$7 \mid 2014$}

Poètes et poésie en révolution

\title{
De la révolution politique à la révolution poétique en passant par la contre-révolution, ou « le moment $1800 »$
}

Gérard Gengembre

\section{OpenEdition}

Journals

Édition électronique

URL : http://journals.openedition.org//rf/1185

DOI : $10.4000 /$ Irf. 1185

ISSN : 2105-2557

Éditeur

IHMC - Institut d'histoire moderne et contemporaine (UMR 8066)

Édition imprimée

Date de publication : 31 décembre 2014

Référence électronique

Gérard Gengembre, « De la révolution politique à la révolution poétique en passant par la contrerévolution, ou « le moment 1800 » », La Révolution française [En ligne], 7 | 2014, mis en ligne le 31 décembre 2014, consulté le 10 décembre 2020. URL : http://journals.openedition.org//rf/1185 ; DOI https://doi.org/10.4000//rf.1185

Ce document a été généré automatiquement le 10 décembre 2020.

(c) La Révolution française 


\title{
De la révolution politique à la révolution poétique en passant par la contre-révolution, ou « le moment 1800 »
}

\author{
Gérard Gengembre
}

« Le mouvement complète ainsi son action.

Grâce à toi, progrès saint, la Révolution Vibre aujourd'hui dans l'air, dans la voix, dans le

livre ;

Dans le mot palpitant le lecteur la sent vivre ;

Elle crie, elle chante, elle enseigne, elle rit.

Sa langue est déliée ainsi que son esprit. »

1 On aura reconnu la Réponse à un acte d'accusation de Victor Hugo, qui précise encore : "J'ai dit aux mots: Soyez république !... »... comme si la Déclaration des droits de l'homme trouvait son équivalent, son alter ego dans la discours poétique même. Et de continuer : «Et quand j'ai vu, parmi la foule qui se rue, / Pendre, par tous les mots que le bon goût proscrit, / La lettre aristocrate à la lanterne esprit. / Oui, je suis ce Danton! je suis ce Robespierre!"... Comme si la Terreur avait été à l'ordre du jour dans les Lettres... Mais cet aujourd'hui, c'est le XIX ${ }^{\mathrm{e}}$ siècle tel que le Romantisme l'a modelé. Or, pour que vive ce vivace et bel aujourd'hui, il a fallu non seulement la révolution politique mais la lente maturation d'une révolution poétique. Force est de constater que celle-ci n'alla pas de soi. «La littérature qui exprime l'ère nouvelle n'a régné que quarante ou cinquante ans après le temps dont elle était l'idiome » C'est ainsi que dans ses Mémoires d'outre-tombe (XIII, 11), Chateaubriand propose une explication liée au décalage chronologique. Le romantisme ne s'impose véritablement qu'avec la Révolution de 1830, et non dans la suite immédiate de 1789. Entre ces deux événements historiques, le conflit littéraire bat son plein, en liaison avec les débats idéologiques. L'accomplissement du « 14 juillet du goût » réclamé dès 1825 par Ludovic Vitet dans le journal libéral Le Globe ne se produit qu'au terme d'une difficile gestation. Or, nous 
aimerions insister sur l'importance de la sensibilité, de la conception, voire de la théorie, et de la posture contre-révolutionnaires dans la maturation complexe de cette révolution poétique ${ }^{1}$.

Il s'agit de nous inscrire dans l'une des questions posées par cette journée d'étude : « explorer les angles morts d'une historiographie plus attentive au néoclassicisme progressiste et aux contre-révolutionnaires (qu'ils soient classiques ou romantiques) qu'aux tentatives d'émancipation simultanément politique et littéraire ». Nous en serons bien d'accord: tout procède de la coupure révolutionnaire. On se contentera de rappeler un facteur bien connu, même si la recherche a encore à découvrir sur ce point : une part notable du romantisme français et de sa poésie sera d'abord fortement teintée par les positions et le sentiment contre-révolutionnaire dont sont porteurs les émigrés. Victimes d'une Histoire tragique, nostalgiques d'un temps et d'une civilisation révolus, déboussolés dans un monde agressivement moderne, les émigrés et les revenants contestent les Lumières jugées responsables du terrible bouleversement. Si les doctrines contre-révolutionnaires jouent un rôle difficilement appréciable dans la constitution du romantisme, il importe de souligner la force du renouveau religieux et spirituel qui les accompagne. Depuis Paul Bénichou, on parle de contre-révolution spirituelle, qui discrédite la figure du Philosophe, privilégie l'émotion dans la poésie et dans l'art, fait s'équivaloir sens littéraire et sens de l'âme. Le catholicisme se redécouvre comme source de réconfort et comme évangélisme, mais également comme institution sociale et comme garant d'ordre et d'autorité. Retour à la foi et renouvellement de la pratique religieuse se voient liés à une glorification plus générale du passé et de ses valeurs. Nostalgie et idéalisation trouvent leur expression dans une poétisation et leur chantre dans une nouvelle version de la figure du poète, chargé de nourrir la mémoire, de consoler, d'émouvoir, en somme d'opposer au présent la puissance de l'imaginaire.

3 De nombreux travaux récents convergent vers la définition d'un moment, d'une conjoncture post-révolutionnaire où se pose avec acuité la question du statut même de la littérature dans une société révolutionnée, et celle de la place et de la fonction de l'écrivain moderne. Les analyses élaborées par Germaine de Staël, par Chateaubriand, par Bonald et par les Idéologues présentent une incontestable commodité: elles occupent des positions stratégiques sur l'échiquier idéologique dès l'époque de la Convention thermidorienne, puis du Directoire et enfin du Consulat, autrement dans cette phase de la Révolution qui voit un affrontement entre gestionnaires des acquis politiques, économiques ou sociaux et survivants du jacobinisme d'une part, et une guerre idéologique ravivée entre ces mêmes gestionnaires et les contrerévolutionnaires d'autre part. Si les Idéologues et le groupe des amis de Germaine de Staël contribuent à l'élaboration d'un libéralisme à la française appuyé sur les valeurs révolutionnaires et républicaines, ils divergent quelque peu sur la question du statut et de la mission de l'homme de lettres de type nouveau. Tout en pensant en termes d'histoire littéraire (autrement dit d'historisation du fait littéraire), Les Idéologues tendent de plus en plus vers un «conservatisme littéraire ». De son côté, Germaine de Staël prend en compte une évolution nécessaire de l'écriture, même si elle reste dans les catégories intellectuelles des Lumières.

4 La doctrine contre-révolutionnaire française s'inscrit dans deux monuments fondateurs, la Théorie du pouvoir politique et religieux de Louis de Bonald (1796), et les Considérations sur la France de Joseph de Maistre (1796 ou 1797). Alors que Maistre 
développe une interprétation providentialiste et ne s'attarde guère sur la réalité des conflits idéologiques, des déterminismes socio-économiques ou des enjeux purement politiques (il ne s'intéresse qu'à la « métapolitique »), Bonald propose une théorisation générale des formes historiques du pouvoir et des lignes de force de l'Histoire. Dans cette perspective générale, il consacre d'importants développements à la philosophie, à la littérature et aux écrivains, développements qui seront systématisés dans les textes publiés autour de 1800 . On y trouve les formulations contre-révolutionnaires les plus cohérentes et les plus systématisées concernant la littérature considérée comme production d'écrits et l'homme de lettres conçu comme homme public.

5 Louis de Bonald représente donc idéalement la position contre-révolutionnaire, et non seulement parce qu'il est l'inventeur de la célébrissime formule «la littérature est l'expression de la société. » Face à lui, Germaine de Staël, dès son ouvrage resté inédit Des Circonstances actuelles qui peuvent terminer la Révolution et des principes qui doivent fonder la république en France (1798) définit une littérature expression des Lumières et d'une Révolution raisonnable, restituée à ses principes fondateurs. Cela aboutira à De la littérature, publié en 1800. L'acte d'écrire n'échappe dès lors plus à sa mise en situation dans un contexte historique, politique, idéologique. Quant à Chateaubriand, on sait qu'il inaugure avec le Génie du christianisme une nouvelle poétique, tant par l'apologie de la religion chrétienne que par les deux récits Atala et René. De là les questions qui me semblent pouvoir être posées: qu'est-ce qu'écrire en 1800, ou plus exactement comment penser l'acte d'écriture ? Quels sont les enjeux ? Les modèles et références ? Quel projet peut-on définir? Quels rapports l'écriture et l'écrivain doivent-ils entretenir avec la société, avec le pouvoir? Quelles liaisons doivent exister entre poétique et politique, liaisons qui doivent rester aussi peu dangereuses que possible?

6 Si le bouleversement révolutionnaire a eu des effets contradictoires (discrédit de l'homme de lettres et nouvelles missions assignées à celui-ci, révolte de l'écriture contre la politique et prise de parti de l'écrivain dans le jeu politique ou le combat idéologique, nouvelle définition de la sensibilité et tentation du repli, etc...), l'évolution de la nation vers un nouvel ordre politico-social oblige un certain nombre d'auteurs et de théoriciens à repenser la mission de l'écrivain de type nouveau, libéré des anciennes contraintes mais soumis à de nouvelles, riche d'une expérience historique complexe, confronté à une situation inédite. Se met en place ce qu'on pourrait appeler une première mouture de l'intellectuel intervenant directement dans le débat politique, élaborant une philosophie de l'écriture, voire du style, pour mieux la définir comme activité sociale.

7 À cet égard, il semble bien que les questions de la poésie et du statut du poète s'inscrivent dans un moment 1800. Celui-ci, pour confus qu'il puisse apparaître, dessine les linéaments d'un XIX ${ }^{e}$ siècle de l'écriture militante parce que nécessairement prise dans les multiples réseaux du pouvoir, lui-même travaillé par les idées. Aucune question relative à l'écriture, à la production d'écrits, à la nature du travail de l'écrivain n'est comprise indépendamment de la conjoncture et de l'avenir national. La littérature devient partie prenante de la réalité nationale, insérée dans son tissu même. Dès lors, 1800 marque la naissance d'une modernité dont tout le siècle sera issu. Le moment 1800 apparaît donc comme l'inscription historique d'une conception sinon radicalement nouvelle mais en tout cas pensée selon des termes nouveaux d'une politique du littéraire et d'une intime liaison entre poétique et politique. La littérature devient de façon claire un produit conditionné par de nombreuses déterminations. Un produit 
certes particulier, mais un produit dépendant d'une histoire et d'une société. Voilà pourquoi $\mathrm{M}^{\mathrm{me}}$ de Staël écrit son De la littérature, l'une des première réflexions sur les conditions sociologiques et historiques de la création littéraire ${ }^{2}$.

On aurait pu penser que l'approche idéologiste de la nouvelle conjoncture littéraire au sortir de la Révolution permettait de définir une conception de la littérature et de la poésie en rapport avec la fonction de l'écrivain : éclairer et émouvoir. Il n'en fut rien malgré les avancées de Cabanis dans sa Lettre à Thurot sur les poèmes d'Homère, où il propose les principes d'une esthétique de l'impression et, à défaut d'une poétique, suggère d'autres recherches. En considérant les productions artistiques du point de vue de leurs effets psychologiques et non plus du beau en soi ou de la conformité à certaines règles, le sensualisme permet un progrès décisif. Peut s'instaurer une esthétique scientifique, qui compléterait l'édifice idéologiste, et rationaliserait le champ épistémologique de l'art. Si tout art est destiné à agir sur tel sens, il importe d'étudier d'abord le sens auquel il se rapporte, la manière dont sont produites les impressions, leur degré, leur persistance, leur nature... Si le beau est ce qui produit une sensation de plaisir, s'instaure une esthétique de l'efficacité. Les conséquences sont immenses : aux qualités de clarté, simplicité, harmonie, susceptibles de toucher surtout l'intelligence, et pièces maîtresses de l'arsenal classique, une telle esthétique ajoute en les privilégiant les éléments propres à susciter le frémissement intérieur. L'esthétique romantique est en germe dans de telles propositions. Certes le beau idéal reste au centre du dispositif, mais il se modifie singulièrement en fonction du rôle attribué à l'imagination et au sentiment, promouvant la perception de l'individuel, du particulier, légitimant même les fécondes notions de sympathie ou d'inconscient ${ }^{3}$. Cependant, l'influence de la position idéologiste reste mineure. C'est du côté des analyses contrerévolutionnaires qu'il nous faut nous tourner en ces années où la poésie occupe une place capitale. Elle s'impose comme l'un des lieux où s'énoncent bilans, aspirations, recentrements. Pour ce qui nous concerne ici, la voix poétique ne doit pas être évaluée selon la qualité de ses discours mais selon les réponses qu'elle apporte à quelques-unes des questions fondamentales posées dans ces années post-révolutionnaires : qu'en est-il du progrès? Du sentiment? Du rêve ? De l'esprit?

\section{Bonald et la mission de la littérature}

9 Essayons de situer, au moins en partie, l'influence de la doctrine contre-révolutionnaire dans la constitution du romantisme. Selon Bonald, considéré comme expression symbolique des structures politiques, religieuses et morales de la société, l'art apparaitt comme instance de détermination des convictions fondatrices. Ce qui implique que Bonald définisse à la fois la référence au beau, l'origine sociale de l'art et sa finalité. On peut parler d'une véritable métaphysique des contradictions chez Bonald pour qualifier sa métaphysique du beau. Le beau contradictoire qui traverse l'individu, la société et l'art reflète la structure du réel. C'est dire que cette contradiction n'est autre que l'écart essentiel entre les domaines spirituel et matériel. Vie / mort, esprit / matière, âme / corps : voilà les couples de termes cruciaux, qui déterminent le beau, le goût et la société elle-même. Disons que l'art est, pour Bonald, le lieu de concentration des tensions qui structurent l'existence des êtres créés.

Si Bonald embrasse la totalité des arts, le primat de la littérature ne ressortit pas seulement à une culture mais à une position que les textes de la Restauration 
expliciteront: tout acte d'écriture se fonde dans le don divin de l'écriture sainte, comme tout acte de langage s'origine dans la révélation divine. "On ne peut s'empêcher de regarder la religion comme la cause première et cachée des différences qu'on remarque dans le style des divers peuples et des diverses écoles de littérature, lorsqu'on observe qu'il y a plus de sentiments et d'images, et par conséquent plus d'éloquence et de poésie, partout où un culte plus sensible offre aux affections de l'homme des motifs plus présents, et à ses sens des objets plus extérieurs; et qu'il y a moins de sentiments et d'images, et même moins d'orateurs et de poètes, là où le culte, dénué d'objets sensibles, n'occupe que le pur intellect ${ }^{4}$.»

11 Bonald s'accorde donc avec Chateaubriand pour célébrer les vertus créatrices issues du christianisme ${ }^{5}$. Modèle inépuisable, le beau religieux se définit aussi comme source du beau. Donc la révélation divine est la condition même du beau. La religion et l'art partagent une finalité commune : la formation morale de la personne humaine. L'une en tant que cause, l'autre comme medium. Bonald tente donc une synthèse entre l'ancien et le nouveau, au profit d'une restauration des valeurs. S'il ouvre la modernité du rapport des sociétés à leurs œuvres littéraires, c'est pour appeler de ses vœux une littérature de régénération. Dans le frémissement romantique il ne veut d'abord percevoir que l'exaltation du beau chrétien, que l'aspiration à un ordre refondé, que le chant de l'âme tournée vers Dieu. S'il accorde à l'écrivain une place éminente, c'est qu'il veut en faire un chantre et un propagandiste, un homme du service public, à la fois un auxiliaire et un prophète de la foi et de la monarchie. C'est dire qu'il prend à son compte l'ambition messianique de l'écrivain romantique, en même temps que sa définition politique. Bonald a voulu un style nouveau au service de la ContreRévolution.

C'est ici qu'intervient ce que l'on appellera une tendance romantisante de l'esthétique bonaldienne. S'il place l'œuvre littéraire dans la lumière idéologique, il met l'accent sur l'expressionnisme. L'écriture procède de la faculté d'imagination créatrice d'êtres fictifs. On ne saurait donc ramener l'art à l'imitation d'une nature préexistante. La production littéraire s'enracine dans l'entrecroisement de la pensée et de l'image pour créer des "paroles figurées" ayant pour fonction de "spiritualiser les images des corps, matérialiser les idées d'êtres intellectuels, idéer les figures ${ }^{6}$.» C'est pourquoi il salue le style émotif déployé par son auteur dans le Génie du christianisme :

Il passe aux harmonies de la religion chrétienne avec nos sentiments, nos souvenirs, nos passions; et cette partie de son ouvrage est empreinte d'une mélancolie douce et rêveuse, qui est le caractère dominant du faire, ou plutôt de l'être même de l'auteur, et qui sans doute n'est que l'impression qui lui est restée de grandes scènes et de grands malheurs, comme le long frémissement que conserve un corps sonore, après le coup qui l'a frappé. Cet auteur a vu aussi les grands contrastes de la vie, et il les a tracés dans ses écrits : il a vu le monde entier n'être que le grand contraste du passé et de l'avenir; partout l'opposition du néant à l'être, le malheur dans le berceau, l'espoir dans le tombeau et dans la mort tout l'intérêt de la vie ${ }^{7}$.

13 En effet, le débat sur la littérature et la foi avait pris encore plus d'ampleur lors du Concordat de 1801. La célébration solennelle de ce dernier coïncida avec la parution de Génie du christianisme, qui consacre en 1802 la poétique de la religion chrétienne, et prépare les voies de la littérature nouvelle. Chateaubriand avait été précédé sur ce chemin de reconquête des esprits et des cœurs par Ballanche et son Du sentiment de 1801. Dès lors, romantisme et christianisme seront longtemps inséparables, avant que romantisme et religion au sens plus général du terme ne forment couple. Le siècle 
semble commencer sous les auspices d'une célébration de la religion de l'homme sensible, conférant à la littérature moderne "ornements graves" et "beautés majestueuses et sombres ${ }^{8}$ ", et la possibilité d'exprimer des "pensées mystérieuses ", des "souvenirs doux et tristes", des "choses graves et élevées ${ }^{9}$ ", ce qui semble bien être la conception bonaldienne de la mission élevée assignée par l'époque à la littérature. Le beau moderne s'identifie aux beautés de la religion: tel serait le Concordat des lettres. L'approbation bonaldienne du romantisme s'installe à l'évidence dans les limites d'une intégration de l'émotivité dans la morale chrétienne et le catholicisme renaissant. L'ordre émotif est subordonné à l'ordre théologique et éthique. Il n'en reste pas moins une vibration particulière de la lyre, particulièrement adéquate à une époque de redécouverte et de refondation. La poétique du romantisme naissant s'inscrit dans la renaissance d'une nation martyrisée par la Révolution.

Non seulement l'écrivain s'impose des devoirs que lui rappellent sans cesse la société et le pouvoir politique, mais il se doit de transporter les lecteurs ou les spectateurs dans un monde idéal qui renvoie à la société l'image de sa perfection toujours en devenir. Il faut donc régenter les écrivains et les soumettre à la prescription des lois sociales et politiques. Dès 1796, dans la Théorie de l'éducation sociale et de l'administration publique qui complète la Théorie du pouvoir, Bonald prévoit le statut des écrivains dans une société régénérée. Censure, éditions expurgées des auteurs célèbres, sévérité des lois appliquées aux écrivains : le programme est en place. Il va de pair avec l'exaltation des valeurs religieuses et morales. La boucle est bouclée : si l'intelligence de l'art et de la littérature est en fin de compte une science politique, elle relève des mêmes principes. D'autant que le rôle de la religion est déterminant. D'où la supériorité de la Bible sur tout autre littérature, et le renouveau nécessaire de la littérature religieuse et chrétienne.

eligion chrétienne constitue le seul véritable progrès dans l'histoire de l'humanité en même temps qu'elle définit une permanence de la nature humaine, susceptible d'être traitée par le littéraire, et donc d'être le sujet même de la poétique. Le christianisme possède en lui-même la capacité de s'adapter à l'avancée historique des lumières et de la res politica, tout en permettant, tout en favorisant la dramatisation littéraire de la passion sous toutes ses formes, de ses modalités et de ses effets. Ceci conduit à dépasser la seule théologie et à lire les œuvres littéraires comme expression des aspirations de l'homme au divin. Il s'agit de recourir à la culture comme preuve du christianisme et aux configurations symboliques allant de la Chute à la mélancolie comme produits d'une énergétique créatrice dynamisée par l'espoir du retour et de la réintégration. La religion donne un cadre, un socle, une origine et une fin au sentiment.

Dans ces conditions, pour qu'une contre-révolution se mette en branle, il reste à l'écrivain d'accomplir pleinement dans une société régénérée sa mission édifiante et sa fonction pédagogique, en des termes qui se rapprochent des positions staëliennes définissant une mission républicaine de l'homme de lettres moderne :

L'écrivain exerce [...] une fonction publique, et même la plus publique de toutes les fonctions, puisque, de son vivant, il peut être lu par un nombre de personnes qu'aucun orateur n'en pourrait rassembler dans un même lieu ; que même, lorsqu'il n'est plus, il continue à parler aux hommes par ses écrits, et que cette instruction, bonne ou mauvaise, peut durer autant que la société ${ }^{10}$.

17 En dernière analyse, Bonald reste prisonnier d'une conception de la religion comme prescrivant une subordination des passions à un devoir moral intransigeant. Celui-ci va de pair avec les exigences de la loi sociale. Toute théorie du fait littéraire, de sa 
production, de ses modalités demeure intrinsèquement liée à l'ordre. Telle se dessine strictement l'enceinte conceptuelle où s'inscrit une tentative de donner à la France en voie de régénération contre-révolutionnaire une assise théorique et surtout une pratique littéraire moderne. Là s'arrête l'apport bonaldien à la modernité. Il reste mince, et, s'il le relativise, ne remet nullement en cause le tableau de la perfection littéraire du Grand Siècle français, dont, de son côté, Mme de Staël ne conteste pas l'inaccessible beauté. Si la nostalgie du Grand Siècle parcourt tous ses textes, il faut convenir que son argumentation tend à définir de manière embryonnaire la nation littéraire. Que toute société se construise autour de facteurs spirituels et matériels qui façonnent l'art conduit Bonald, sinon à interroger, du moins à suggérer la spécificité de la nation, même s'il s'agit d'en glorifier la constitution. Tout se passe comme si les prémices théoriques se métamorphosaient en une ébauche de théorisation autre. L'exemplarité française apparaît autant dans l'absolu classique que dans la dégénérescence $d u X^{X V I I I}$ siècle, et la mission de l'homme de lettres se définit désormais comme celle d'un homme du service public, auxiliaire et prophète de la foi et de la monarchie.

Ainsi, en dépit d'une conception de la littérature qui la contextualise et la considère analogiquement à l'idéologie et à la structure d'une société, Bonald ne peut dépasser le simple rapport de correspondance, ou, si l'on préfère de réflexion entre les déterminations et la production. Quelles que soient les intrications de ces facteurs, Bonald en revient toujours à quelques principes universels. Ainsi le fondateur de la sociologie et de la politique du littéraire, du moins par sa géniale formule, se trouve-t-il condamné à laisser à d'autres le soin d'exploiter ce gisement intellectuel. Il n'en reste pas moins que Bonald a bien inventé une nouvelle manière de comprendre la littérature.

\section{Progrès, Religion, Génie}

Philosophiquement, la contre-révolution dénonce l'illusion du progrès. Notons que la recension faite par Fontanes du De la littérature de Germaine de Staël va dans le même sens. Fontanes s'en prend surtout à l'idée de progrès. Que l'histoire de l'humanité soit marquée par le progrès et que, par conséquent, l'ère moderne soit supérieure aux époques antérieures, est pour lui une chimère. Le progrès se limite à la science et aux techniques, mais la vraie création ressortit à la littérature: «Il faut le dire: les créations les plus brillantes et les plus durables sont celles de l'éloquence et de la poésie $^{11}$. " La raison en est que leur pouvoir se fonde sur les "passions humaines", lesquelles «ne changent pas. » Dans la morale, les arts et la politique, il n'existe pas de progrès, et d'ailleurs, dans ces domaines, il n'y a rien de nouveau à découvrir. C'est une erreur que d'aborder la vie humaine à coups de calculs et de raisonnements. «Lorsque tout est soumis à des analyses philosophiques, tout perd de son charme.» Le " charme » des choses réside selon Fontanes dans leur « mystère »; l'« âme » ne se plaît que dans " des sentiments mystérieux et infinis ». Celui qui est sensible à « la gloire, la vertu et la beauté ", ferait mieux de laisser de côté les "doctrines qui dessèchent le cœur et l'imagination. » L'on commet une erreur stupide à "glorifier les sciences au détriment des beaux-arts. » Dans sa louange de la poésie et de l'imagination, Fontanes reçoit l'appui de Chateaubriand, dont il était ami depuis l'émigration. Chateaubriand traitait des mêmes thèmes, mais mettait plus l'accent sur la foi. Parce que «seul le 
christianisme a compris l'homme, [...] j'attribue à la foi tout ce que Madame de Staël attribue à la philosophie ${ }^{12}$. "Seul le christianisme avait apporté quelque intelligence dans l'univers des sentiments humains, tous les grands écrivains avaient été des chrétiens et, toujours selon Chateaubriand, tout écrivain qui refusait de croire, bannissait "l'infini » de son œuvre. Or, au lieu de "progrès » et de "perfectibilité ", c'était cet « infini », mystère éternel, qui importait réellement. Le " génie » n'était rien d'autre que d'en avoir conscience.

Le but de Chateaubriand était de démontrer que le christianisme était «la plus poétique et la plus humaine » de toutes les religions et qu'il était la religion la plus favorable "aux arts et aux lettres. »Celle-ci fut présentée comme une composante d'une alliance entre la littérature et le catholicisme. À la manière de Fontanes, Chateaubriand construisit une antinomie entre littérature et science, antinomie qui l'emportait sur tout et dont le premier terme représentait le sentiment et la passion, et le second la froide raison. Cette dichotomie en recoupait toute une série d'autres, où la foi était du côté de la littérature, des sentiments, de la morale et de l'humanité, alors que la science appartenait à la sphère de la raison, de l'incroyance, de la froideur et même du crime. La science et la philosophie constituent, dit encore Chateaubriand, une menace pour les poètes et les écrivains, mais non moins pour la vie sentimentale, pour l'Église et pour toute la société.

21 Chateaubriand replace l'histoire de la littérature et ses rapports avec la nation et la société à la lumière du christianisme. En considérant la solitude de l'homme moderne, il accorde à la littérature, particulièrement dans le cas de l'épopée et de la tragédie chrétiennes, une valeur anthropologique. Grâce au merveilleux chrétien, à la poésie chrétienne, se pense une littérature à la fois nationale et personnelle. L'esthétique classique reste une référence, voire un modèle, mais en fait elle se voit mise au service d'un art nouveau, où la double poésie du songe et de la vision occupe une place décisive. De là aussi la place de la Bible considérée comme "Livre des livres ", réservoir de symboles et de mythes fondateurs. Clerc, le poète doit devenir aussi prêtre et prophète. Chanter le vague des passions revient à dire la perte de l'idée de Dieu et par conséquent d'une société bien ordonnée. L'individu déboussolé appartient à un monde désorganisé. La nostalgie va de pair avec un projet de société, lui-même lié à un projet de civilisation. Il faut revenir à la transcendance, véritable fondement des lois. Alors la liberté sera bien comprise et deviendra effectivement le champ de l'activité sociale. En somme, les rapports sociaux doivent être sacralisés. Voilà pourquoi le père Aubry est le véritable héros d'Atala.

Durant les quelques années qui suivent la prise de pouvoir de Napoléon, un groupe d'intellectuels ralliés autour du Mercure de France de Fontanes se démarque en préconisant le sentiment et le pouvoir d'imagination comme sources principales de la littérature. Celles-ci étaient présentées comme une alternative aux modèles intellectuels dominants et avaient un caractère ouvertement antiscientifique et antirationaliste. Contre la prétention de la raison, Chateaubriand et ses amis défendent tout ce qui échappait au calcul et à la compréhension. Echanger la philosophie contre la foi, la raison contre le sentiment et le savoir scientifique contre l'enchantement des miracles et des mystères: c'est ainsi que naît une conception différente de la littérature. Dans la prose exubérante de Chateaubriand, les idéaux du classicisme furent bientôt ruinés. Au "goût » succède le « génie ». La littérature est identifiée à la poésie, non plus à de claires et gracieuses représentations de la réalité, mais à une 
recréation lyrique de tout ce qui existait. L'écrivain n'est plus un homme de lettres, expression qui devint fade et quelque peu suspecte, mais un visionnaire et un troubadour. Pour Chateaubriand, la réalité est énigmatique, obscure et étourdissante, autant dire inexplicable. Vouloir comprendre la création n'est que vaine ambition, et pur orgueil que de prétendre pouvoir la comprendre.

Tous les premiers romantiques français insistent d'une manière ou d'une autre sur le fait qu'il importait de ne pas analyser ni examiner la nature, mais de la chanter et de s'étonner de sa beauté et de ses secrets éblouissants. À l'opposé des choses terrestres et finies, ils recherchent l'envoûtement du «mystère », de l'« inépuisable », de l'« infini », et ils confient à l'écrivain la mission de s'en faire le témoin. Ainsi, la position contrerévolutionnaire érige la figure du poète comme truchement de la spiritualité, comme chantre de la religion, comme consolateur des âmes. Si révolution poétique il y a, c'est dans la mélodie du style, dans sa capacité à émouvoir, à toucher, à faire vibrer. Certes, il ne s'agit encore que des moyens rhétoriques bien compris et poussés au bout de leur possibilités musicales, et il n'est nullement question de mettre quelque bonnet que ce soit au dictionnaire. Et le poète peut louer le philosophe, le doctrinaire contrerévolutionnaire, à l'instar de Lamartine dédiant à Bonald son poème Le Génie de 1817 :

Ainsi des sophistes célèbres / Dissipant les fausses clartés, / Tu tires du sein des ténèbres / D'éblouissantes vérités. / Ce voile qui des lois premières / Couvrait les augustes mystères, / Se déchire et tombe à ta voix ; / Et tu suis ta route assurée, / Jusqu'à cette source sacrée / Où le monde a puisé ses lois.

Or cette question du Génie est bien l'une de fondements de la posture romantique. Si le cas Chateaubriand est flagrant, d'autres poètes des premières années du siècle offrent l'exemple de cette vocation de la poésie à dire les nouveaux enjeux et tracer les nouvelles perspectives. Et cela s'énonce d'abord par une dénonciation des passions politiques, mauvaises, délétères, ou bien un ralliement la politique consulaire, même chez des auteurs plus proches des Lumières. Voyez Chênedollé (1769-1833) et son Génie de l'Homme, publié à Paris en 1807 chez H. Nicolle, avant de reparaitre en 1812 (avec un "Avertissement ») puis chez Gosselin en 1822. Reprenant le programme de l'Hermès de Chénier, Chênedollé, en même temps qu'un Delille (Les Trois Règnes de la Nature, 1808), donne avec ce poème un bon échantillon de la production poétique impériale, férue de grands genres, et attentive à orner de ses vers le savoir. Voulant appliquer la poésie aux sciences, il tente ainsi d'apparaitre comme un Lucrèce moderne et d'accomplir une mission éducative autant qu'esthétique. Dans l'Avant-propos, Chênedollé trace luimême le plan de son ouvrage: "J'ai montré l'homme d'abord étudiant les cieux; ensuite cherchant à connaître le séjour qu'il habite ; puis se repliant sur lui-même, et s'efforçant de deviner sa propre nature; enfin se considérant comme être social, et cherchant quelle forme de gouvernement donne la plus grande mesure de biens, et présente la plus petite quantité d'inconvénients.» Fortement teinté d'idéologie concordataire, le poème ne manque jamais de louer Dieu, de donner les preuves de son existence et de révérer la "puissante nature ", " fille du grand Être ». L'hommage final rendu à l'ordre impérial constitue l'acmé de cette élévation :

Déjà l'esprit humain qui, fier de sa vieillesse / y avait, dans les transports d'une orgueilleuse ivresse / Voulu refaire l'Homme et la Société, / De ses propres excès lui-même épouvanté, / De la perfection abjure la manie, / Et partout dans l'Etat rétablit l'harmonie. / Pour raffermir enfin ses pensers inconstans, / Il invoque aujourd'hui l'autorité des tems ; / Déjà l'expérience, à la voix éternelle, /

À repris les États sous sa noble tutelle. / Tout ce qui fut utile est encore honoré. / Les temples sont rendus à leur culte sacré, / Et la Religion, qui s'accroît de ses 
pertes, / Voit ses solennités avec pompe r'ouvertes. / La Politique errante, et sans guide et sans art, / Qu'on vit, dix ans entiers, gouverner au hasard, / Sur la Religion maintenant appuyée, / Et forte des secours d'une telle alliée, / De nos calamités va terminer le cours.

Ce refus de la politique, ou plutôt ce ralliement au pouvoir compris comme réconciliateur, traduit un besoin d'ordre, de refondation. Il s'agit d'exprimer les dimensions sentimentale et spirituelle de l'individu moderne. Si un Millevoye par exemple peut se livrer à la déploration lyrique dans ses Élégies de 1811 et apparaître comme une voix pré-lamartinienne en chantant les souffrances d'un Moi meurtri par la perte ou hanté par la mort («La Chute des feuilles », «Le Poète mourant »), ou bien annoncer le genre troubadour, avec «Le Festin de la châtelaine ", des ballades et des romances, il vient en quelque sorte donner figure au poète tel que le souhaite la réaction contre-révolutionnaire : enchanter en chantant. Il serait évidemment abusif de verser au seul crédit de la contre-révolution les évolutions de la conception de la poésie autour de 1800. Il n'en reste pas moins que son idéologie a joué un rôle décisif. Cette aube du XIX ${ }^{e}$ siècle se lève sur un espace du littéraire mal délimité. Serait-ce cette indécision qui contraint les théoriciens à tracer des limites et des chemins, à arpenter les territoires de l'âme, de l'esprit et du cœur, à poser des points de repère ? Jamais une telle conjoncture ne s'était dessinée. La Révolution fut bien l'un de ces moments climatériques de l'Histoire, dont l'impact sur la littérature s'est d'abord fait sentir par la remise en cause de la stratégie d'auteur et du statut même de l'écrit. Tout se passe comme si, pour s'accomplir, la révolution poétique romantique avait dû en passer par un moment contre-révolutionnaire afin de poser la figure du poète en s'opposant.

\section{NOTES}

1. Cette contribution n'oublie pas que le romantisme et la révolution poétique ne sont pas des idées purement françaises. En ce-qui concerne l'idée de la révolution esthétique et poétique, elle est, déjà dans les années 1790, développée chez Immanuel Kant et de manière encore plus profonde chez Friedrich Schiller («Über die ästhetische Erziehung des Menschen »).

2. J'ai repris ici un développement paru dans le Bulletin de la Société Chateaubriand, $n^{\circ} 43,2001$.

3. Voir Gérard GENGEMBRE, «L'esthétique des Idéologues et le statut de la littérature ", dans Hans Heinz HoLz, alii. (dir.), Die französische Revolution: Philosophie und Wissenschaften, Bd. I, Annalen der internationalen Gesellschaft für dialektische Philosophie Societas Hegeliana, VI, Milano, Edizioni Guerini e Associati, 1989.

4. Louis de BONALD, «Du style et de la littérature ", article paru dans le Mercure de France, 30 août, 13 décembre et 20 décembre 1806, dans Louis de Bonald, CEuvres choisies, Tome I, Écrits sur la littérature, Gérard Gengembre et Jean-Yves Pranchère (éd.), Éditions Classiques Garnier, 2010, p. 142.

5. La communauté de vision semble à ce point fondatrice qu'elle impressionne les chroniqueurs de l'époque ; ainsi le Journal de l'Empire du 14 mars 1807 publie-t-il sous la signature « N » : « Trois auteurs principaux, Madame de Staël, M. de Chateaubriand, M. de Bonald, dans des ouvrages très remarquables, ont appliqué, depuis quelques années, à la littérature, ce grand principe dont $\mathrm{M}$. de Bonald a créé l'expression : La littérature est l'expression de la société. » 
6. Louis de BONALD, Législation primitive, 1802, dans Euvres complètes, édition Migne en 3 volumes, 1859-1864, tome I, p. 1157.

7. Louis de BONALD, Génie du christianisme, 1802, recension parue dans John CONLEY, L'Esthétique sociale de Bonald, Dissertation en vue de l'obtention du grade de docteur en philosophie, Université catholique de Louvain, 1988, vol. 2, p. 485-486.

8. Ibid., p. 485.

9. Ibid., p. 486.

10. Louis de BonALD, «Des jeunes écrivains », article paru dans La Gazette de France, $1^{\text {er }}$ décembre 1810, dans Euvres choisies, Tome I, Écrits sur la littérature, op. cit., p. 344.

11. Louis de FONTANES, «De la littérature, considérée dans ses rapports avec les institutions sociales, par madame de Staël-Holstein », Mercure de France, $1^{\mathrm{er}}$ messidor an 8 (20 juin 1800), $\mathrm{n}^{\circ} 1$, p. 13-27, et $1^{\text {er }}$ thermidor an 8 (20 juillet 1800$), n^{\circ} 3$, p. 171-196.

12. François-René de CHATEAUBRIAND, «Lettre au comte de Fontanes, sur la seconde édition de l'ouvrage de $\mathrm{M}^{\mathrm{me}}$ de Staël », Mercure de France, $1^{\mathrm{er}}$ nivôse an 9 (22 décembre 1800), n 13, p. 14-38.

\section{RÉSUMÉS}

Autour de 1800, l'idéologie contre-révolutionnaire a joué un rôle décisif dans l'évolution de la poésie et surtout de sa conception. Elle réagit sous l'impact de la Révolution sur la littérature, lequel s'est d'abord fait sentir par la remise en cause de la stratégie d'auteur et du statut même de l'écrit. En témoignent, par exemple, les réflexions théoriques de Bonald, Chateaubriand ou Fontanes comme les poèmes de Millevoye ou de Chênedollé. Tout se passe comme si, pour s'accomplir, la révolution poétique romantique avait dû en passer par un moment contrerévolutionnaire afin de poser la figure du Poète en s'opposant.

Around 1800, French counter-revolutionary thinkers play a significant part in the questioning of the mission and content of poetry after the Revolution, which radically transformed the position and status of the author and his works. See for example Bonald, Chateaubriand, Fontanes or such poets as Millevoye or Chênedollé. It looks like, to be effective, the romantic revolution in poetry had to go through a counter-revolutionary phase, as if an ideological opposition was necessary to impose the Poet as a modern figure.

\section{INDEX}

Mots-clés : Bonald, Contre-Révolution, Génie, poésie, rReligion 